\title{
Endemic visceral leishmaniasis in a patient from lower Monferrato hills in provence of Alessandria, Italy
}

\author{
Luigi Di Matteo',Andrea Pagetto², Clara Lazzara',Andrea Rocchetti' \\ I SSD Microbiologia, Dipartimento di Patologia Clinica, ASO "SS. Antonio e Biagio e Cesare Arrigo" Alessandria \\ 2 SC Medicina Interna, Dipartimento Internistico, ASO “SS. Antonio e Biagio e Cesare Arrigo", Alessandria
}

Key words: Leishmaniasis, Pancytopenia, Piemonte, Leishmania, Diagnosis

\section{Leishmaniosi viscerale autoctona in un paziente nella provincia di Alessandria (Basso Monferrato), Italia}

\section{SUMMARY}

Visceral leishmaniasis is endemic in Italy as well as in the entire Mediterranean area and Campania is the italian region in which most cases occur. Dog leishmaniasis, common along the coast from Liguria to Southern Italy, is nowadays reported in areas as far inland as Monferrato hills, the outskirts of Turin and lake Garda southern shores.

Mr. B.B. (male 67 years old) came almost by chance to the attention of our Medical department for acute fever and pancytopenia; further symptoms comprised subacute weight loss, splenic and limph node enlargment, an ulcerative lesion on the right arm. The patient appeared to be unavoidably destined to splenectomy until a Leishmania antibody test was requested to our laboratory. The assay was performed using an immunocromathographic method for specific IgM and by immunofluorescence to determine the total antibody titre towards Leishmania. Microscopic examination (Giemsa stain) and PCR were also performed to look for Leishmania infantum amastigotes in blood, bone marrow and skin.

All tests, except microscopic examination, corroborated a Leishmania infection, and a dramatical clinical improvement was seen with Amphothericin B treatment.

We conclude that an accurate anamnesis is mandatory if the Laboratory must be perform a rational diagnosis.

\section{INTRODUZIONE}

La leishmaniosi viscerale (LV), antropozoonosi, è diffusa in varie aree del mondo (India, Pakistan, Cina, Medio Oriente, America Latina, Sudan ed Etiopia) con circa 500000 mila nuovi casi all'anno. L'era AIDS (anni '85-'90) ha permesso di definire che la LV è endemica in Italia ed in tutto il bacino del mediterraneo.

La regione in cui si verifica il maggior numero di casi umani (50-60) è la Campania seguita dalla Sicilia. Attualmente si sta diffondendo anche in territori del Nord Italia come le colline del Monferrato e del torinese nonché sul versante meridionale del Lago di Garda (14). La leishmaniosi canina è diffusa dalla Liguria a tutto il Centro-Sud Italia con prevalenze medie del 1215\% (con picchi che superano il 40\%) (7).

Il protozoo parassita, inoculato in forma flagellata (promastigote) da ditteri ematofagi di diversi generi, viene rapidamente fagocitato dai mononucleati, perde il flagello (amastigote) e parassita gli organi ricchi di fagociti tessutali. L'uomo è l'ospite definitivo accidentale e, in alcuni casi, anche il serbatoio $(2,6)$. L'incubazione dura tra i due e gli otto mesi ma anche anni ed è favorita da situazioni di immunodepressione (neoplasie infezione HIV, corticoterapie o terapie antirigetto).

Nel paziente normoergico la LV può essere asintomatica o paucisintomatica, si manifesta più frequentemente nei bambini o negli anziani ed è frequentemente scambiata per malattia emolinfoproliferativa.

La malattia é caratterizzata da febbre spesso irregolare con puntate di tipo settico alternate a periodi di remissione che possono anche simulare una risposta alla terapia antibiotica. Frequenti sono perdita di peso e pallore marcato.

Un segno clinico tipico è la epato-splenomegalia. Si osservano, inoltre, leucopenia, piastrinopenia, innalzamento della VES, ipoalbuminemia con aumento delle gamma globuline e moderata elevazione delle transaminasi $(8,12)$.

La diagnosi è diretta con tecniche microscopiche, colturali e di biologia molecolare e indiretta con tecniche immunologiche che possono essere facilmente eseguite presso tutti i laboratori di micro-

\section{Corresponding author: Luigi Di Matteo}

Via Ugo Foscolo 21 - 15067 Novi Ligure (AL)

Tel. Lab.: 0I3I 207440 - Cell.: 3472574780

E-mail: luigi.di-matteo@laposte.net - E-mail: Lab.Idimatteo@ospedale.al.it 
biologia. Per una diagnosi completa è opportuno rivolgersi a centri specializzati quali l'Istituto Superiore di Sanità, il Laboratorio di Parassitologia dell'IRCCS San Matteo di Pavia o l'Istituto di Medicina Veterinaria dell'Università di Milano.

In questo lavoro gli autori descrivono un caso umano di leishmaniosi osservata nel basso Monferrato nella provincia di Alessandria, sebbene l'infezione sia stata verosimilmente contratta in Liguria, ove sono stati segnalati casi umani (16).

\section{CASO CLINICO}

Uomo di 67 anni, B.B., viene ricoverato nel 2010 presso la Struttura Complessa di Medicina Interna del Dipartimento Internistico dell'Azienda Ospedaliera di Alessandria. L'anamnesi patologica remota rivela: iperplasia prostatica benigna (IPB), pregresso attacco ischemico transitorio (TIA), Morbo di Paget, intervento protesico all'anca, ernio-plastica bilaterale e gastroresezione per ulcera peptica. L'anamnesi patologica prossima evidenzia un malessere generalizzato, accompagnato da febbre irregolare, astenia, calo ponderale e sudorazione notturna che si manifesta dopo un soggiorno estivo ad Arenzano (Liguria) nel 2009. Il paziente esegue esami ematochimici (globuli bianchi $3200 / \mathrm{mcl}$; piastrine $86000 / \mathrm{mcl}, \mathrm{Hb} 9.6 \mathrm{~g} / \mathrm{dl}$ ) e sulla base del referto viene sottoposto a visita ematologica ed a successivi accertamenti: esofagogastroduodenoscopia (EGSD), colonscopia, tomografia assiale computerizzata (TAC) del torace-addome e biopsia osteomidollare (BOM) da cui emerge un quadro di midollo ipercellulare con iperplasia trilineare, dismielopoiesi, plasmocitosi reattiva con immunofenotipo non monoclonale B. L'ipotesi diagnostica è quella di un paziente complesso affetto da una malattia linfoproliferativa. La presenza di pancitopenia ingravescente induce a considerare un intervento di splenectomia e viene ricoverato nel reparto di Medicina Interna perdurando le cattive condizioni cliniche. Il medico internista esegue un accurato esame obiettivo che rivela la presenza di una lesione a placca, eritematosa a fondo ulcerato, non dolente, sulla cute del braccio destro che il paziente riferisce essere la trasformazione di una papula comparsa dopo la puntura di un insetto durante il soggiorno al mare ad Arenzano. Questi dati anamnestici, associati ad un quadro elettroforetico caratterizzato da una importante ipergammaglobulinemia, inducono il clinico a richiedere al laboratorio di Microbiologia una serie di accertamenti volti a diagnosticare una eventuale eziologia parassitaria sostenuta da Leishmania.

\section{MATERIALI E METODI}

\section{Test rapido per la Leishmania}

È stato eseguito il Leishmania Strip Quick Test
(Cypress Diagnostics, Belgium), un immunodosaggio qualitativo per la rilevazione di anticorpi specifici verso l'antigene ricombinante $\mathrm{k} 39$ di Leishmania su siero (4).

\section{Test di immunofluorescenza indiretta (IFA) per leishmaniosi}

Il test IFA per la rivelazione di anticorpi IgG umani rivolti verso antigeni di promastigoti della Leishmania tropica consente di determinare il titolo anticorpale utilizzando diluizioni seriali del siero in esame. Nel test Leishmania IFA (MarDx Diagnostics $^{\mathrm{R}}$, Trinità Biotech Company, Carlsbad, $\mathrm{Ca})$ la preparazione dell'antigene è costituita da proamastigoti di L. tropica coltivati su terreno tripla-N (9). Un quantitativo standardizzato di parassiti lavati viene poi messo nei pozzetti di vetrini da microscopio appositamente preparati. Il siero dei pazienti è posto nei pozzetti ricoperti di antigene dove gli anticorpi, se presenti, si legano all'antigene. La reazione viene visualizzata tramite l'uso di un coniugato, costituito da IgG (specifico della catena gamma) di capra, marcato con FITC e purificato per affinità. Il coniugato, osservato con un microscopio a fluorescenza, si lega agli anticorpi umani attaccati alle cellule provocando la fluorescenza dei trofozoiti.

\section{Esame microscopico}

L'esame microscopico permette di evidenziare la presenza di amastigoti all'interno di macrofagi intralesionali o in sede extracellulare. L'indagine è stata eseguita su campioni di aspirato midollare e da frammenti bioptici prelevati dall'ulcera e dai linfonodi satelliti.

\section{PCR convenzionale}

Il Dna di Leishmania è stato amplificato utilizzando una coppia di primers RV1 e RV2 che rappresentano sequenze di basi complementari alla sequenza bersaglio contenuta nel DNA di $L$. infantum. Questo test molecolare è stato eseguito secondo la metodica descritta da Lachaud su sangue midollare e periferico e sulle biopsie (17).

\section{RISULTATI}

L'esame citologico eseguito mediante l'esame microscopico dei preparati colorati con Giemsa risultava negativo anche dopo attenta e scrupolosa osservazione. La sierologia era positiva sia al test immunocromatografico che in immunoflorescenza con titolo anticorpale elevato (1:5120). La PCR qualitativa per la ricerca del DNA di $L$. infantum risultava positiva su tutti i materiali analizzati.

\section{DISCUSSIONE}

Per raggiungere la diagnosi di leishmaniosi i dati 
anamnestici, i segni clinici eventualmente presenti ed i risultati delle prove di laboratorio devono essere integrate tra loro.

La PCR, la sierologia e, solo in alcuni tessuti, la microscopia dopo colorazione Giemsa hanno tutte una elevata sensibilità (13). Se la citologia e l'istologia risultano negative la sierologia acquista un'importanza fondamentale per considerare il soggetto malato.

La sensibilità e la specificità dell'IFA sono prossime al $100 \%$ (1) e per tale motivo questo test viene considerato il metodo sierologico di riferimento (15). Il titolo anticorpale, nel caso risulti elevato (1:640), indica che il soggetto è affetto da leishmaniosi mentre se il titolo risulta basso non è possibile diagnosticare con certezza la malattia senza ricorrere ad una PCR su biopsia cutanea e su midollo osseo (10).

Il test immunocromatografico, di semplice e rapida esecuzione, può risultare utile nel contesto degli accertamenti laboratoristici in quanto la buona sensibilità $(90 \%)$ potrebbe essere sfruttata in prima battuta per orientare la diagnosi.

Il caso descritto esprime la complessità del percorso clinico strumentale per giungere ad una corretta diagnosi di leishmaniosi quando l'insieme dei sintomi e dei segni presenta numerosi elementi confondenti.

In effetti l'ipotesi diagnostica iniziale, sulla base degli accertamenti ematologici (quadro di midollo ipercellulare con iperplasia trilineare, dismielopoiesi, plasmocitosi reattiva con immunofenotipo non monoclonale B), era stata quella di un paziente affetto da una malattia linfoproliferativa.

Solo un successivo ed accurato esame obiettivo (presenza di una lesione sulla cute del braccio destro comparsa dopo puntura di insetto al mare) ha indotto il clinico a riconsiderare il tracciato elettroforetico caratterizzato da una evidente ipergamma-globulinemia non monoclonale $\mathrm{B}$, indirizzando il sospetto verso una patologia infettiva sostenuta da Leishmania evitando la splenectomia.

$\mathrm{Si}$ evidenzia come sempre vada effettuata un'accurata anamnesi ed uno scrupoloso esame obiettivo specialmente in presenza di sintomi aspecifici che inducono a ipotizzare patologie a carico del sistema reticolo-endoteliale, in quanto si possono scorgere quegli elementi predittivi di leishmaniosi che, seppur rara nelle forme sintomatiche, rappresenta una realtà in aree endemiche sempre più estese. Si ricorda come una corretta identificazione di specie o meglio una classificazione zimodemica, possibile solo dopo isolamento colturale, siano fondamentali per una corretta epidemiologia e permettano talvolta di localizzare il luogo dove è avvenuta l'infezione.

\section{BIBLIOGRAFIA}

1. Ashford DA, Bozza M, Freire M, et al. Comparison of the polymerase chain reaction and serology for the detection of canine visceral leishmaniasis. $\mathrm{Am} \mathrm{J}$ Tropical Med Hyg 1955; 53 (3): 251-5.

2. Boelaert M, Croft SL, Desjeux P, et al. Visceral leishmaniasis: current status of control, diagnosis and treatment and a proposed research and development agenda. Lancet Infect Dis 2002; 2: 494-501.

3. Castagnaro M, Crotti A, Fondati A, et al. Leishmaniosi canina: linee guida su diagnosi, stadiazione, terapia, monitoraggio e prevenzione. Veterinaria 2007; 21 (3): 19-32.

4. Chianura L, Cirasino L. Leishmania infection in a 51year-old woman with sarcoidosis: case report. APMIS 2006; 114 (11): 825-7.

5. David CV, Craft N. Cutaneous and mucocutaneous leishmaniasis. Dermatol Ther 2009; 22 (6): 491-502.

6. de Carneri I. Parassitologia Generale Umana, Casa Editrice Ambrosiana, 13th edn. Milano, 2004.

7. Ferroglio E, Maroli M, Gastaldo S, Mignone M, Rossi L. Canine Leishmaniasis, Italy. Emerg Infect Dis 2005; 11: 1618-20.

8. Gaeta GB. Clinica della leishmaniosi viscerale nell'adulto: cambiano le modalità di presentazione? Parassitologia 2004; 46: 225-6.

9. Gatti S, Gramigna M, Klersy C, et al. Diagnosis of visceral leishmaniasis: the sensitivies and specificities of traditional methods and a nested PCR assay. Annals of Tropical Medicine and Parasitology 2004; 98: 667-76.

10. Gradoni L, Gramiccia M. Leishmaniasis. In: OIE manual standards for diagnostic tests and vaccine. $4^{\text {th }}$ ed. Officine International des Epizooties, Paris, France, 2000; 803-12.

11. Herwaldt BL. Leishmaniasis. Lancet 1999; 354 (9185): 1191-9.

12. Kemp M, Theander TG. Leishmaniasis. Ugeskr Laeger 2000; 162 (46): 6203-7.

13. Lachaud L, Marchergui-Hammani S, Chabbert E, et al. Comparison of six PCR methods using peripheral blood for detection of canine visceral leishmaniasis. $J$ Clin Microbiol 2002; 40 (1): 210-5.

14. Lanotte G, Rioux JA, Maazoun R, et al. Biology and Epidemiology. In: Peters W \& Killick-kendrick R, eds. The Leishmaniases in Biology and Medicine. London: Academic Press, 1987; 1: 1-120.

15. Mettler M, Grimm F, Capelli G, Camp H, et al. Evaluation of enzyme-linked immunosorbent assays, an immunofluorescent-antibody test, and two rapid tests (immunochromatographic-dipstick and gel tests) for serological diagnosis of symptomatic and asymptomatic Leishmania infections in dogs. J Clin Microbiol 2005; 43: 5515-9.

16. Neuber H. Leishmaniasis. J Dtsch Dermatol Ges 2008; 6 (9): 754-65.

17. Nicolle $\mathrm{CH}$. Culture du parasite de Bouton d'Orient. Comptes Rendu Hebdomadaire des Sci de l'Acad des Sci Paris 1908; 146: 842-3.

18. Palombo E. Current treatment for cutaneous leishmaniasis: a review. Am J Ther 2009; 16 (2): 178-2.

19. Robert LJ, Handman F, Foote SJ. Leishmaniosis. BMJ 2000; 321: 801-4.

\section{RINGRAZIAMENTI}

Un ringraziamento va al Dr. Michele Mortarino del Dipartimento di Patologia Animale, Igiene e Sanità Pubblica Veterinaria per aver eseguito la parte di biologia molecolare. 